\title{
Measurement of Adenylate Cyclase Activity in the Minute Bovine Ciliary Epithelial Cells During the Pharmacological Stimulation of Adrenergic and Cholinergic Receptors
}

\author{
Norifumi Sawada, ${ }^{1}$ Atsushi Sugiyama, ${ }^{1 *}$ Kenji Kashiwagi, ${ }^{2}$ \\ Shigeo Tsukahara, ${ }^{2}$ and Keitaro Hashimoto ${ }^{1}$ \\ ${ }^{1}$ Department of Pharmacology, Yamanashi Medical University, Tamaho, Yamanashi, Japan \\ ${ }^{2}$ Department of Ophthalmology, Yamanashi Medical University, Tamaho, Yamanashi, Japan
}

\begin{abstract}
Although essential to the secretion of aqueous humor, little is known about the signal transduction underlying postreceptor adrenergic and cholinergic processes in the ciliary epithelium. We adopted a highly sensitive fluorometric assay technique in order to examine adenylate cyclase activity in minute membrane preparations made from the bovine ciliary epithelial cells. The protein concentration of the preparation was $3-5 \mathrm{mg} /$ $\mathrm{ml}$. Norepinephrine $\left(10^{-7}, 10^{-6}\right.$ and $\left.10^{-5} \mathrm{M}\right)$ and carbachol $\left(10^{-7}\right.$ and $\left.10^{-5} \mathrm{M}\right)$ were incubated with $10 \mu$ of membrane preparation to analyze the extent of the receptor-coupled influences on the adenylate cyclase activity. Meanwhile, forskolin $\left(10^{-5} \mathrm{M}\right)$ was used to estimate the maximum adenylate cyclase activity. After the initial enzymatic destruction of noncyclic adenine nucleotides and phosphorylated metabolites, the diester linkage of cyclic AMP was cleaved and then
\end{abstract}

converted to ATP. The ATP was enzymatically amplified to about 10,000 times of fructose-6-phosphate. The NADPH, formed when the fructose-6-phosphate was converted to 6-phosphogluconolactone, was measured fluorometrically. Basal and forskolin-stimulated maximum adenylate cyclase activities ( $\mathrm{pmol} / \mathrm{mg}$ protein $/ \mathrm{min})$ were $29.6 \pm 7.6$ and $86.6 \pm 7.2$ (mean \pm SE), respectively. Norepinephrine increased the adenylate cyclase activity in a dose-dependent manner, while carbachol hardly affected the activity. These results indicate that the adenylate cyclase activity can be measured in the minute ciliary epithelial cells and, moreover, that the current assay can be applied to assess the efficacy of newly available ophthalmic solutions or systemic drugs influencing adenylate cyclase activity in a discrete portion in the eye. J. Clin. Lab. Anal. 13:9094, 1999. (1) 1999 Wiley-Liss, Inc.

Key words: adenylate cyclase; bovine ciliary epithelial cell; cyclic AMP; enzymatic fluorometric assay; nonradioactive

\section{INTRODUCTION}

Since intraocular pressure (IOP) is a function of the balance between fluid input and drainage out of the globe, the strategies for the treatment of open-angle glaucoma fall into two classes: reduction of aqueous humor secretion and enhancement of aqueous outflow (1-8). Cholinomimetics, $\alpha$ agonists and $\beta$-blockers have been found to be useful in reducing IOP, and the clinical application of these agents has been well established in patients with glaucoma (1-8). However, little is known about the signal transduction underlying postreceptor adrenergic and cholinergic processes in a discrete portion in the eye, partly due to the lack of a versatile yet sensitive assay of adenylate cyclase activity for the minute tissue mass (9-11).

To begin to overcome this difficulty, we adopted a recently developed, highly sensitive, nonradioactive fluorometric assay technique to examine the effects of pharmacological interventions on the adenylate cyclase activity in minute membrane preparation made from the bovine ciliary epithelial cells $(12,13)$. In this study, norepinephrine and carbachol were incubated with the membrane preparations to analyze the extent of the receptor-coupled influences on the regional adenylate cyclase activity, while forskolin was used to estimate the maximum adenylate cyclase activity.

Grant sponsor: Japanese Ministry of Education; Grant numbers: 09771416 and 09770058 .

*Correspondence to: Dr. Atsushi Sugiyama, Department of Pharmacology, Yamanashi Medical University, Tamaho, Nakakoma Yamanashi, 409-3898, Japan. E-mail: atsushis@res.yamanashi-med.ac.jp

Received 29 September 1998; Accepted 16 November 1998 


\section{MATERIALS AND METHODS}

All experimentation was performed according to the rules and regulation of the Committee for Research at the Yamanashi Medical University.

\section{Production of the Membrane Preparation}

Ciliary epithelial cells were obtained from six different bovine eyes to prepare respective membrane preparations as previously described (14). Briefly, a circumferential incision was made in the sclera $8 \mathrm{~mm}$ behind the limbus. The anterior portion of the globe, including anterior sclera, lens, iris, and ciliary body were excised and placed on a culture dish. The ciliary body was excised and positioned with its posterior surface upward, and washed with $\mathrm{Ca}^{2+}-\mathrm{Mg}^{2+}$ free-Hank's solution (D-Hank's, Gibco, Grand Island, NY). The ciliary body was separated from sclera, placed in another culture dish with its inner surface facing down, and immersed in $0.25 \%$ trypsin solution for $2 \mathrm{hr}$ at $37^{\circ} \mathrm{C}$. The ciliary epithelium was then separated from the ciliary body under the dissecting microscope. The epithelium was homogenized in 5 volume of icecold SET buffer (0.25 M sucrose, $0.1 \mathrm{mM}$ EDTA, $5.0 \mathrm{mM}$ Tris-HCl, $\mathrm{pH} 7.4$ ) and centrifuged at 10,000g for $5 \mathrm{~min}$ (13). The pellet was resuspended in 5 volume of SET buffer and the mixture was centrifuged under the same setting. After this procedure was repeated twice, the pellet was finally suspended in 4 volume of SET buffer. The protein analysis was performed using commercially available protein assay reagent (Pierce, Rockford, IL). The membrane suspension in a concentration of 3.0-5.0 $\mathrm{mg}$ of protein $/ \mathrm{ml}$ was stored at $-80^{\circ} \mathrm{C}$ until enzyme activity was measured.

\section{Measurement of Adenylate Cyclase Activity}

The adenylate cyclase activity of the membrane preparation made from the ciliary epithelium was measured using an enzymatic fluorometric assay technique $(12,13)$. The assay consists of two parts: the production of cAMP by the adenylate cyclase (Part A), and the measurement of newly formed cAMP (Part B). Each reaction is summarized in Figure 1.

\section{Part A}

Ten $\mu \mathrm{L}$ of adenylate cyclase mix (100 mM Tris-acetate, pH 7.4; $20 \mathrm{mM} \mathrm{KCl} ; 10 \mathrm{mM} \mathrm{MgCl}_{2} ; 20 \mathrm{mM}$ phosphoenolpyruvate; 2 mM ATP; $20 \mu \mathrm{M}$ GTP; 2 mM dithiothreitol; $0.04 \%$ bovine serum albumin; $0.1 \mathrm{mM} 3$-isobutyl-1methylxanthine (IBMX); $100 \mu \mathrm{g} / \mathrm{mL}$ pyruvate kinase) was added to each microcentrifugation tube in duplicate with or without either of forskolin, carbachol or norepinephrine. Next, the membrane suspension in a volume of $10 \mu \mathrm{L}$ was added to each tube. The reaction mixture and membrane suspension, both before and after being combined, must be maintained at $0^{\circ} \mathrm{C}$ to ensure the same starting time for all assay tubes. The reaction was initiated by placing the tubes in a water bath maintained at $37^{\circ} \mathrm{C}$. After $20 \mathrm{~min}$, the reaction was terminated by heating at $90^{\circ} \mathrm{C}$ for $5 \mathrm{~min}$. The mixture was vortexed 3 times and centrifuged at 10,000g for $5 \mathrm{~min}$. The supernatant solution was stored for the following cAMP assay.

Part A:

ATP $\stackrel{\text { adenylate cyclase }}{\longrightarrow}$ CAMP $+2 P_{i}$

Part B:

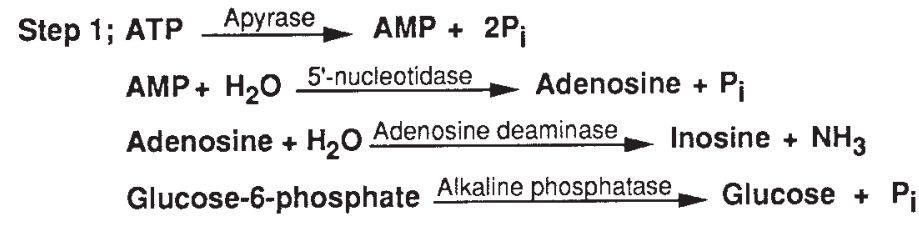

Step 2; cAMP $\stackrel{\text { Phosphodiesterase }}{\longrightarrow}$ AMP

AMP + ATP (trace) Myokinase 2 ADP

ADP + Phosphoenolpyruvate Pyruvate kinase ATP + Pyruvate

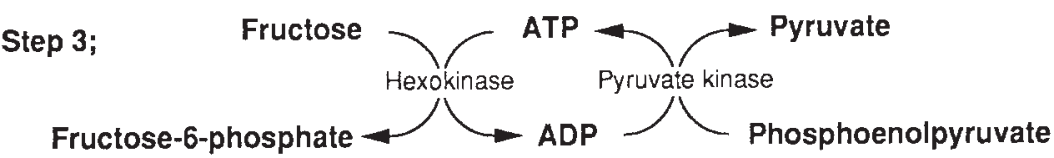

Step 4; Fructose-6-phosphate $\stackrel{\text { Phosphoglucoisomerase }}{\longrightarrow}$ Glucose-6-phosphate

Glucose-6-phosphate + NADP+ Glucose-6-phosphate dehydrogenase

6-Phosphogluconolactone + NADPH + $\mathrm{H}^{+}$

Fig. 1. Diagram of reactions in the enzymatic fluorometric assay for cyclic AMP (cAMP). $P_{i}$ : inorganic phosphate. 


\section{Part B}

A volume of $5 \mu \mathrm{L}$ of the supernatant was transferred to a $10 \times 75 \mathrm{~mm}$ disposable assay tube (Iwaki Lab Ware, Tokyo, Japan) in duplicate. For the cAMP standard, $5 \mu \mathrm{L}$ of known amount of cAMP was added to the tubes. The cAMP assay consists of the following four steps: (1) a volume of $25 \mu \mathrm{L}$ of reaction mix A (100 mM Tris-HCl, pH 8.0; 2 mM MgCl $2 ; 2$ units/mL apyrase; 2.5 units $/ \mathrm{mL} 5^{\prime}$-nucleotidase; 10 units $/ \mathrm{ml}$ adenosine deaminase; 20 units $/ \mathrm{mL}$ alkaline phosphatase) was added to each tube. The mixture was incubated at $37^{\circ} \mathrm{C}$ for $1 \mathrm{hr}$. Enzymes were then destroyed by heating for $10 \mathrm{~min}$ at $90^{\circ} \mathrm{C}$; (2) a volume of $25 \mu \mathrm{L}$ of reaction mix B (100 mM Tris-HCl, $\mathrm{pH} \mathrm{8.0;2} \mathrm{mM}$ $\mathrm{MgCl}_{2} ; 0.01 \%$ bovine serum albumin; 12 munits/mL phosphodiesterase; $150 \mathrm{mM} \mathrm{KCl} ; 2 \mathrm{mM}$ dithiothreitol; $80 \mathrm{nMATP} ; 3 \mathrm{mM}$ phosphoenolpyruvate; 4.5 units $/ \mathrm{mL}$ myokinase; 80 units $/ \mathrm{mL}$ pyruvatekinase) was added to each tube from step 1 . The mixture was incubated for $8 \mathrm{hr}$ at room temperature; (3) a volume of $25 \mu \mathrm{L}$ of reaction mix C (100 mM Tris- $\mathrm{HCl} \mathrm{pH} \mathrm{8.0;2} \mathrm{mM}$ $\mathrm{MgCl}_{2} ; 0.01 \%$ bovine serum albumin; $3 \mathrm{mM}$ fructose; 60 units/ $\mathrm{mL}$ hexokinase) was added to the tubes from step 2 on ice. The mixture was incubated for $2 \mathrm{hr}$ at $37^{\circ} \mathrm{C}$; and (4) a volume of 125 $\mu \mathrm{L}$ of reaction mix D (100 mMTris-HCl, $\mathrm{pH}$ 8.0; 3.2 mM EDTA; $0.6 \mathrm{mM} \mathrm{NADP}{ }^{+}, 1$ unit $/ \mathrm{mL}$ phosphoglucoisomerase; 0.5 unit/ $\mathrm{mL}$ glucose-6-phosphate dehydrogenase) was added to the tubes from step 3.After the mixture was incubated for $20 \mathrm{~min}$ at room temperature, a volume of $600 \mu \mathrm{L}$ of distilled water was added to each tube. The concentration of NADPH was measured as an emission fluorescence of $460 \mathrm{~nm}$ by the $340 \mathrm{~nm}$ of excitation wave using fluorometric spectrophotometer (Hitachi, F3000, Tokyo, Japan). The sensitivity of the fluorometer was set such that a reading of 1 fluorometric unit was equivalent to $100 \mathrm{pmol}$ of NADPH in $900 \mu \mathrm{L}$ of Tris-HCl buffer (50 mM, pH 8.0).

\section{Biochemicals}

Carbachol was obtained from Wako Chemical Industries (Osaka, Japan), while 5'-nucleotidase, apyrase, phosphoenolpyruvate, phosphodiesterase, IBMX, sucrose, fructose, dithiothreitol, norepinephrine, and forskolin were obtained from Sigma Chemical Company (St. Louis, MO). All other enzymes and substrates were obtained from BoehringerMannheim Company (Indianapolis, IN).

\section{Statistical Analysis}

The results of adenylate cyclase measurements from each individual experiment are expressed as mean $\pm \mathrm{SE}$. The statistical comparisons of mean values were evaluated by paired $t$-test or by one-way, repeated measures ANOVA followed by Contrasts. A $P$-value less than 0.05 was considered significant.

\section{RESULTS}

\section{Sensitivity and Specificity of the Assay}

A representative standard curve of cAMP is shown in Figure 2. The standard curve has a slope of 2.4 and fluorescence

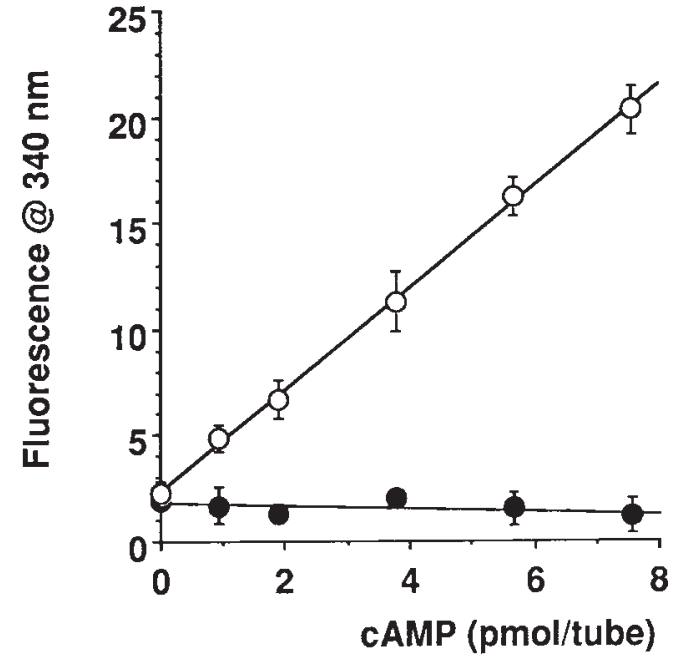

Fig. 2. A representative standard curves of cAMP assayed in the presence (open circles) and absence (close circles) of phosphodiesterase in step 2 . The equation for the cAMP standard curve is $\mathrm{y}=2.4 \mathrm{x}+2.4$. Ten fluorescence units are equivalent to $1 \mathrm{nmol} /$ tube of NADPH. Data are expressed as mean $\pm \mathrm{SE}$.

readings from 2.4 to 20.3 units over cAMP concentration range from 0 to $7.6 \mathrm{pmol} / \mathrm{tube}$. When the step 2 was carried out without phosphodiesterase, the standard curve became almost flat with a slope of 0 and average fluorescence reading of 1.8 (closed circles), indicating that the increase of the fluorescence readings (open circles) specifically depends on the added cAMP. In addition, the ratio of the range for fluorescence readings over the background noise was about $10(\mathrm{n}=$ 6), as shown in Figure 2, indicating the high $\mathrm{S} / \mathrm{N}$ ratio of the current assay.

\section{Stability of Membrane Preparations}

The stability of membrane preparations was initially assessed over $40 \mathrm{~min}$. The linearity between the cAMP production and the incubation time is shown in Figure 3. Since the production rate of cAMP in the absence and presence of forskolin $\left(10^{-5} \mathrm{M}\right)$ was constant up to $20 \min (\mathrm{n}=6)$, the incubation time of $20 \mathrm{~min}$ in the Part A was chosen to determine the adenylate cyclase activity (cAMP pmol/mg protein/ $\mathrm{min})$ in the following experiments.

\section{Pharmacological Interventions of the Membrane Preparation}

The results of the adenylate cyclase activities by each pharmacological intervention are summarized in Figure 4. The actual adenylate cyclase activities (cAMP pmol/mg protein/min, $\mathrm{n}=6$ ) in the basal and forskolin-stimulated membrane preparations were $29.6 \pm 7.6$ and $86.6 \pm 7.2$, respectively. A significant difference was detected between these two values.

Norepinephrine increased the adenylate cyclase activity in a dose-dependent manner. The actual adenylate cyclase activities 


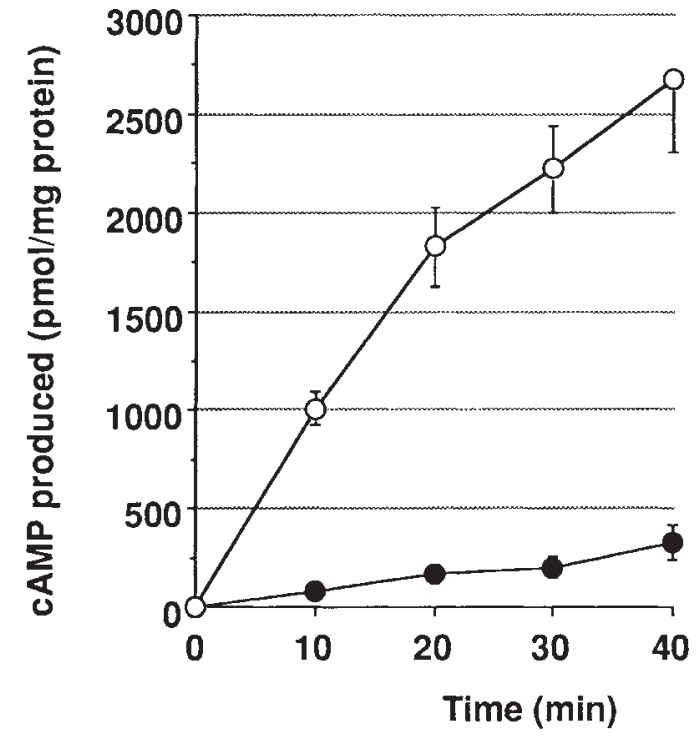

Fig. 3. The stability of adenylate cyclase activity in the membrane preparation made form the bovine ciliary epithelial cells $(n=6)$. For this study a volume of $10 \mu \mathrm{L}$ of adenylate cyclase mix (100 mM Tris-acetate, $\mathrm{pH} 7.4 ; 20$ $\mathrm{mM} \mathrm{KCl} ; 10 \mathrm{mM} \mathrm{MgCl}_{2} ; 20 \mathrm{mM}$ phosphoenolpyruvate; $2 \mathrm{mM}$ ATP; $20 \mu \mathrm{M}$ GTP; 2 mM dithiothreitol; $0.04 \%$ bovine serum albumin; $0.1 \mathrm{mM} 3$-isobutyl-1-methylxanthine (IBMX); $100 \mu \mathrm{g} / \mathrm{mL}$ pyruvate kinase) and $10 \mu \mathrm{L}$ of the membrane preparation were incubated without (closed circles), or with $10^{-5} \mathrm{M}$ of forskolin (open circles). The reaction was linear for at least 20 min. Data are expressed as mean $\pm \mathrm{SE}$.

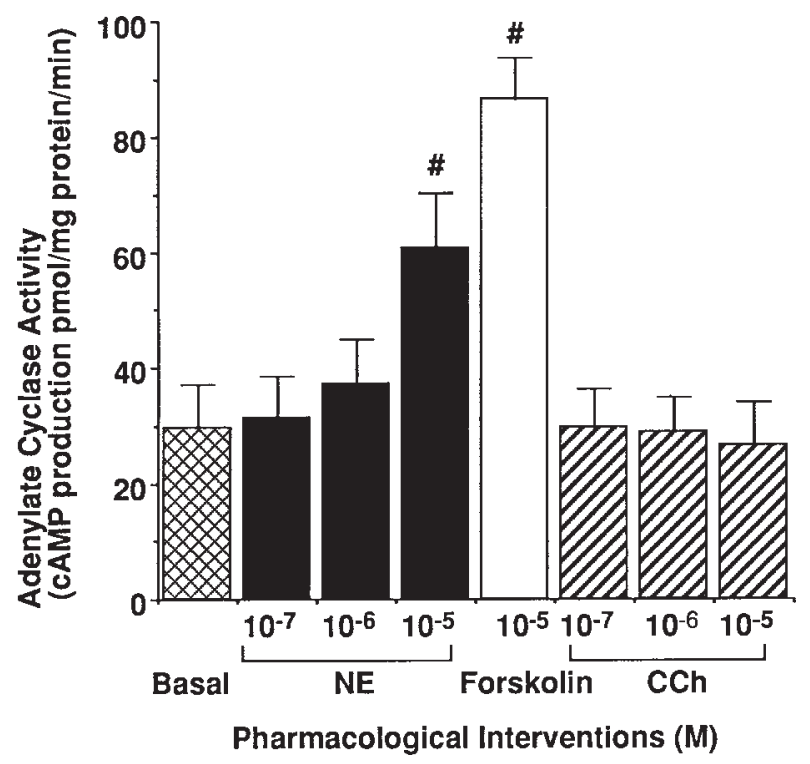

Fig. 4. Summary of the results of adenylate cyclase activity during pharmacological interventions $(n=6)$. The basal adenylate cyclase activity (meshed rectangle) and those with norepinephrine (closed rectangles), forskolin (open rectangle), and carbachol (hatched rectangles) are shown. Data are presented as mean $\pm \mathrm{SE}$. NE, norepinephrine; $\mathrm{CCh}$, carbachol; \#, $P$ $<0.05$ vs. basal value.
(cAMP pmol/mg protein/min, $\mathrm{n}=6$ ) with norepinephrine in concentrations of $10^{-7} \mathrm{M}, 10^{-6} \mathrm{M}$, and $10^{-5} \mathrm{M}$ were $31.3 \pm 7.4$, $37.1 \pm 7.8$, and $60.8 \pm 9.5$, respectively. The adenylate cyclase activity with the highest concentration of norepinephrine was $70.3 \%$ of the maximum activity treated with forskolin.

The actual adenylate cyclase activities (cAMP pmol/mg protein/min, $\mathrm{n}=6$ ) with carbachol in concentrations of $10^{-7}$ $\mathrm{M}, 10^{-6} \mathrm{M}$, and $10^{-5} \mathrm{M}$ were $29.7 \pm 6.5,28.7 \pm 6.1$, and 26.6 \pm 7.3 , respectively. The adenylate cyclase activity tended to be decreased by carbachol in a dose related manner; however, no statistically significant difference was detected among the basal and any of the activities treated with carbachol.

\section{DISCUSSION}

Since the classical assay of adenylate cyclase activities including radioimmunoassay and Salomon method are not sensitive enough to examine a small amount of tissue like ciliary epithelial cells, we adopted a recently developed enzymatic fluorometric assay technique for this study $(12,13)$. The fluorometric assay has been demonstrated to possess a number of potential advantages: the risks and costs associated with radioactivity are eliminated; expense on cost/assay basis are approximately $90 \%$ less than those associated with a radioimmunoassay; no overnight incubation or counting of radioactivity is necessary so the time required from start to finish is significantly shorter when compared with the Salomon assay; and the standard curve is linear and assay sensitivity can be easily varied over a wide range of cAMP $(12,13)$. As demonstrated in the current results, absolute values of adenylate cyclase activities can be measured in a small amount of membrane preparation made from the bovine ciliary epithelial cells, which has not been reported elsewhere as far as we have examined. Thus, the assay can be considered to be applied for assessing the net function of the cAMP related signal transduction system in the ciliary epithelial cells. However, it should be noted that the results of this in vitro assay cannot always reflect the physiological regulation of autonomic nervous system in vivo.

As demonstrated in this study, norepinephrine increased the adenylate cyclase activity in a dose-dependent manner, while carbachol did not affect the activity. These results are in good accordance with the previous reports $(15,16)$, in that the signal transduction system via $\beta$-adrenoceptors may play an important role for the production of aqueous humor, while cholinergic process cannot significantly affect the secretion in the ciliary epithelial cells. Moreover, our results with carbachol are at least in part in accordance with the previous knowledge (8) that cholinergic stimulation decreases the IOP via the increase of outflow of the aqueous humor through the trabecular meshwork rather than the inhibition of the inflow from the ciliary epithelial cells.

In the previous report using the cynomolgus monkey eyes (3), intracameral administration of norepinephrine or epineph- 
rine in a dose of $10 \mu \mathrm{g}$, which was approximately corresponded to initial intracameral concentration of $10^{-4} \mathrm{M}(3,17)$, significantly increased the total outflow facility of aqueous humor, while smaller doses of these drugs hardly affected it. Meanwhile, as shown in this study, the adenylate cyclase activities were stimulated by $10^{-5} \mathrm{M}$ of norepinephrine in the membrane preparation made from the ciliary epithelial cells, which was about ten times lower than that could affect the outflow facility. Thus, it is quite important to clarify the signal transduction system in a discrete portion in the eye to better understand the mechanisms of the drug action. It is noteworthy that we have recently further modified the fluorometric adenylate cyclase assay $(18,19)$. By coupling the assay with a microanalytical method, the volume of membrane preparation can be reduced to $1 \mu \mathrm{L}$ for each experiment in the Part A.

In summary, the current study suggests that the absolute value of adenylate cyclase activity can be measured in the ciliary epithelial cells without using special cAMP antibodies or radioactivity, and that $\beta$-adrenoceptor-dependent pathway, rather than the cholinergic process, may play a significant role in the secretion of aqueous humor. The current assay may be used to assess the efficacy of newly available ophthalmic solutions or systemic drugs influencing $\beta$-adrenoceptors in the eye. Thus, the current approach promises to become a new and potentially important way to assess the mechanisms underlying the effects of catecholamine, adenosine, vasopressin, and other cAMP dependent processes on the anatomically complex tissues in the eye.

\section{ACKNOWLEDGMENTS}

This work was supported in part by the Grant Aid of Japanese Ministry of Education No. 09771416 (K.K.) and No. 09770058 (A.S.). The authors thank Miss Yuko Ishida for her skillful technical assistance.

\section{REFERENCES}

1. Allen RC, Epstein DL. Additive effect of betaxolol and epinephrine in primary open angle glaucoma. Arch Ophthalmol 1986;104:1178-1184.

2. Townsend DJ, Brubaker RF. Immediate effect of epinephrine on aque- ous formation in the normal human eye as measured by fluorophotometry. Invest Ophthalmol Vis Sci 1980;19:256-266.

3. Kaufman PL. Epinephrine, norepinephrine, and isoproterenol dose-outflow facility response relationships in cynomolgus monkey eyes with and without ciliary muscle retrodisplacement. Acta Ophthalmol 1986;64:356-363.

4. Robinson JC, Kaufman PL. Effects and interactions of epinephrine, norepinephrine, timolol and betaxolol on outflow facility in the cynomolgus monkey. Am J Ophthalmol 1990;109:189-194.

5. Bill A. Early effects of epinephrine on aqueous humor dynamics in vervet monkeys (Cercopithecus ethiops). Exp Eye Res 1969;8:35-43.

6. Caprioli J, Sears M, Mead A, et al. Adenylate cyclase stimulation and intraocular pressure reduction by forskolin analogues. J Ocul Pharmacol 1989;5:181-187.

7. Bartels SP, Lee SR, Neufeld AH. The effects of forskolin on cAMP, intraocular pressure and aqueous humor formation in rabbits. Curr Eye Res 1987;6:307-320.

8. Hoffman BB. Adrenoceptor antagonist drugs. In: Katzung BG, editor. Basic and clinical pharmacology. Stamford: Appleton \& Lange; 1998. p 136-151.

9. Crawford KS, Gange SJ, Gabelt BT, et al. Indomethacin and epinephrine effects on outflow facility and cyclic adenosine monophosphate formation in monkeys. Invest Ophthalmol Vis Sci 1996;37:1348-1359.

10. Liu JHK, Gallar J. In vivo cAMP level in rabbit iris-ciliary body after topical epinephrine treatment. Curr Eye Res 1996;15:1025-1032.

11. Tsukahara S, Maezawa N. Cytochemical localization of adenyl cyclase in the rabbit ciliary body. Exp Eye Res 1978;26:99-106.

12. Sugiyama A, Lurie KG. An enzymatic fluorometric assay for adenosine 3':5'-monophosphate. Anal Biochem 1994;218:20-25.

13. Sugiyama A, McKnite S, Lurie KG. Measurement of adenylylcyclase activity with an enzymatic fluorometric assay. Anal Biochem 1995;225:368-371.

14. Hu DN, McCormick SA, Ritch R, Pelton-Henrion K. Studies of human melanocytes in vitro: isolation, purification and cultivation of human uveal melanocytes. Invest Ophthalmol Vis Sci 1993;34:2210-2219.

15. Caprioli J, Sears M. The adenylate cyclase receptor complex and aqueous humor formation. Yale J Biol Med 1984;57:283-300.

16. Jumblatt JE, North GT, Hackmiller RC. Muscarinic cholinergic inhibition of adenylate cyclase in the rabbit iris-ciliary body and ciliary epithelium. Invest Opthalmol Vis Sci 1990;6:1103-1108.

17. Kaufman PL. Isoproterenol dose-outflow facility relationships in vervet monkey. Curr Eye Res 1985;4:877-883.

18. Sugiyama A, McKnite S, Wiegn P, Lurie KG. Measurement of cAMP in the cardiac conduction system of rats. J Histochem Cytochem 1995;43:601-605.

19. Sugiyama A, McKnite S, Adkisson W, Lurie KG. Measurement of adenylylcyclase activity in the AV nodal region of the canine heart: evidence for inhibition by adenosine and acetylcholine. J Cardiovasc Pharmacol 1997;29:734-739. 